\title{
Parturition mode recommendation and symptoms of pelvic floor disorders after obstetric anal sphincter injuries
}

\author{
Matthieu van der Vlist ${ }^{1}$ (D) Daniella Oom ${ }^{1} \cdot$ Joost van Rosmalen ${ }^{2} \cdot$ Astrid van Ovost $^{3} \cdot$ Cornelis Hogewoning $^{1}$
}

Received: 23 September 2019 / Accepted: 23 January 2020

(C) The International Urogynecological Association 2020

\begin{abstract}
Introduction and hypothesis Our primary objective was to evaluate parturition mode (PM) recommendations following obstetric anal sphincter injuries (OASIs) and adherence to these recommendations and to evaluate recurrence of OASIs in women who had a subsequent vaginal delivery (VD). The hypothesis was that adherence to the PM recommendations leads to a reasonable OASI recurrence rate.

Methods This was a retrospective observational cohort study of patients with previous OASIs between 2010 and 2016. After an outpatient visit including 3D transperineal ultrasound to screen for pelvic floor and anal sphincter injuries, all patients received recommendations for a subsequent PM. Patients were invited to complete validated questionnaires 2 to 5 years post-OASIs.

Results The majority of invited patients (265/320) attended follow-up, with 264 receiving a recommendation for PM. Only 5.6\% did not adhere to the received recommendation. One hundred sixty-one patients delivered again, 58\% had a VD, and $42 \%$ had a cesarean section (CS). Recurrence of OASIs was observed in $4.3 \%$ of the patients that had a VD. Fecal incontinence occurred in $4.9 \%$, however any form of anal incontinence in $48 \%$ of patients. While dyspareunia was common in patients with residual external anal sphincter (EAS) injuries and levator ani muscle (LAM) avulsions, anal pain occurred more frequently in EAS injuries and fecal incontinence in LAM avulsions.

Conclusions This study showed that the vast majority of patients followed PM recommendations, and this resulted in a low recurrence of OASIs with a high CS rate. Fecal incontinence after OASIs was correlated with the degree of OASIs.
\end{abstract}

Keywords Obstetric anal sphincter injury $\cdot$ Levator ani muscle avulsion $\cdot$ Anal incontinence $\cdot$ Obstetric deliveries $\cdot$ Cesarean section $\cdot$ Directive counseling

\section{Introduction}

The reported incidence of obstetric anal sphincter injuries (OASIs) after vaginal birth varies widely, ranging from $0.3 \%$ to $>6 \%$, and is dependent upon multiple maternal, fetal and obstetric factors [1-5]. Higher rates of OASIs are reported after operative vaginal delivery (OVD); the incidence varies

Matthieu van der Vlist

m.y.vandervlist@asz.nl

1 Regional Pelvic Floor Unit, Department of Gynecology, Albert Schweitzer Hospital, Albert Schweitzerplaats 25, 3318, AT Dordrecht, The Netherlands

2 Department of Biostatistics, Erasmus Medical Centre, Rotterdam, The Netherlands

3 Erasmus University Rotterdam, Rotterdam, The Netherlands from $2.0 \%$ in multiparous women delivering by vacuum extraction with an episiotomy to $27 \%$ in primiparous women delivering by forceps delivery without an episiotomy [6]. The evidence for mediolateral episiotomies at the time of OVD decreasing the risk of OASIs is less clear with conflicting evidence from two studies [7, 8]. Other risk factors for the development of OASIs are fetal occipital posterior position, fetal macrosomia and increasing maternal age [7, 9]. With a subsequent delivery after OASI, the risk of recurrent OASI is increased by a factor of $1-5[4,10]$.

OASI is a major risk factor for symptoms of anal incontinence-with symptoms often progressing with increasing age [11]. Patients with complaints of anal incontinence shortly after OASIs are likely to have persistent damage of the external anal sphincter (EAS) [12]. Women with one OASI are at increased risk of fecal incontinence after 6 months compared with women without OASI (17 versus 8\%) [13], whereas another study shows no significant difference in anal 
incontinence after 4 years in a small group (16 versus $10 \%$ ) [14]. A recurrent OASI increases the risk of fecal incontinence even further, and the rate nearly doubles (13 versus 24\%) [15]. Long-term follow-up at an average age of 52 years old shows anorectal complaints in $61 \%$ of women after OASI compared with $22 \%$ in women without OASIs. Symptoms worsen with increasing age with only $38 \%$ of women at 42 years of age reporting anorectal complaints [11].

Currently, no randomized controlled trials to address the optimal mode of delivery for subsequent pregnancies following OASI have been undertaken [4]. A case series by Jordan et al. in women who sustained a previous OASI showed no differences in St. Mark's incontinence scores [16] after vaginal delivery (VD) compared with elective cesarean section (CS) when using a standardized protocol to recommend for mode of subsequent delivery [10]. Our hypothesis is that adherence to the parturition mode (PM) recommendation leads to an OASI recurrence rate in line with the experiences of other centers $[10,17]$.

Our primary objective was to evaluate PM recommendations for subsequent deliveries following OASIs and adherence to these recommendations and to evaluate recurrence of OASIs in women who had a subsequent VD. Secondary objectives were to evaluate symptoms of urinary and anal continence, dyspareunia and defecatory dysfunction in the first 5 years after the index injury.

\section{Methods}

\section{Patient selection and study design}

This was a retrospective observational cohort study of all patients with primary OASIs repaired in their index VD at a large teaching hospital in The Netherlands between 2010 and 2016. Patients were selected from the surgical registration system based on the OASI procedure code. No exclusion criteria were used. In The Netherlands homebirths are common practice with patients with low risk pregnancies delivered under supervision of a first-line midwife in primary care, at home or in the hospital. In secondary care deliveries of higher risk patients are in a hospital setting, attended by either a second-line midwife or a medical doctor.

\section{Follow-up}

The patients were evaluated in the outpatient pelvic floor clinic 6 months after delivery or in the subsequent pregnancy. The outpatient clinic visit consisted of a consultation including physical examination and a 3D transperineal ultrasound to screen for residual lesions of the anal sphincter and the levator ani muscle (LAM). These procedures were conducted by one of three different experienced urogynecologists.
Patients received a recommendation on the PM in case of a subsequent pregnancy. This recommendation was given after counseling about risk of recurrence and discussion of the findings from their consultation. Recommendations for VD were classified as "without preconditions" or "with preconditions." Preconditions were defined as no risk-increasing features such as symptoms of anal incontinence in a subsequent pregnancy, fetal macrosomia, an occiput posterior presentation or an eventual instrumental delivery for a subsequent birth. An elective CS was recommended in patients with severe anatomical residual damage of the LAM or sphincter regardless of symptoms of anal incontinence and in patients with anal incontinence symptoms without residual injury of the sphincter. In cases where it was unclear which recommendation could best be given, no preferential recommendation was given.

\section{Data collection}

Characteristics of the index delivery (i.e., during which the OASIs occurred), subsequent delivery and pelvic floor clinic visit were obtained from the electronic patient records. These included the degree of the laceration, mode of delivery, birthweight, episiotomy use, anal incontinence symptoms, PM recommendations, actual mode of delivery and recurrence of OASIs in the subsequent pregnancy. Notes from the surgical repair were used in the classification of the sphincter injuries. The degree of the laceration was scored according to the RCOG classification system of OASIs [18].

For the 3D transperineal ultrasound a Toshiba SSA-660A XARIO was used with a $2.8-7.2-\mathrm{MHz}$ probe. Anal sphincter imaging was performed with the patient in supine resting position. The axial images of the anal canal were visualized at three levels: the subcutaneous, superficial and deep regions of the EAS. A sphincter defect was determined as a discontinuity in the texture of the EAS or IAS. For the assessment of the LAM integrity, an image through the plane of minimal hiatal dimensions at maximum contraction was visualized. LAM avulsion was determined when a unilateral or bilateral discontinuity was seen at the attachment of the puborectal part of the pelvic floor at the pubic bone. Interpretations of these images from the patient files were available for analysis.

All patients were sent a questionnaire by mail to evaluate the symptoms of pelvic floor disorders, 2 to 5 years after OASIs. If the questionnaire was not returned, patients received a telephone reminder. This questionnaire consisted of questions about their obstetric history and questions from a validated Dutch translation, including the Urogenital Distress Inventory (UDI) [19, 20], Defecatory Distress Inventory (DDI) [21] and Incontinence Impact Questionnaire (IIQ) $[19,20]$. The UDI assesses urogenital symptoms, and the DDI assesses the presence of defecation symptoms. The IIQ assesses the impact of incontinence symptoms on daily life. Dyspareunia was considered present if a patient answered 
affirmatively to the question: Do you experience pain during intercourse? The degree of bother caused by dyspareunia was registered using a 4-point Likert scale (1, not at all bothered; 4, bothered quite a bit). Symptom scores were calculated in the domains of urinary incontinence, fecal incontinence, flatus incontinence, pain related to defecation and dyspareunia. The scores of these domains vary between 0 and 100. A high score on a particular domain indicates more bothersome symptoms. The St. Mark's (Vaizey) fecal incontinence severity score was calculated; it grades the severity of anal incontinence on a scale of $0-24$ [16]. Subsequently, we examined to what extent the sphincter defects, LAM defect and classification of OASIs relate to the symptoms of pelvic floor disorders that were obtained from the questionnaires.

\section{Analysis}

Data were summarized using means and standard deviations for normally distributed variables, medians and interquartile ranges (IQRs) for continuous variables that were not normally distributed and using percentages for categorical variables. The 95\% confidence intervals of proportions were calculated with the Wilson score method. The difference in the proportion of patients that adhered to the recommendation of the PM in the VD compared with the CS group were calculated using the chi-square test. Pelvic floor injuries and the grade of OASIs were compared with symptoms of pelvic floor disorder scores in all patients that visited the clinic and returned the questionnaire using the Mann-Whitney and the KruskalWallis tests. All statistical tests were two-sided with a significance level of 0.05 . For statistical analysis we used SPSS 24.0.

The study was approved by the local research ethics commission with approval no. 2012/36.

\section{Results}

\section{Patient selection}

A total of 320 patients were selected from the surgical registration system. This included all patients with OASIs between 2010 and 2016, out of a total of 14,493 vaginal deliveries [derived from the Dutch Perinatal Registry (Perined)], resulting in a $2.2 \%$ OASI rate for the index delivery. A summary of the patient characteristics is shown in Table 1.

\section{Parturition mode recommendation}

After OASIs, 265 patients (83\%) attended follow-up (see flowchart in Fig. 1). The median time between OASIs and clinic visit was 6 months, as per protocol. In 188 patients (71.2\%) a VD and in 71 patients (26.9\%) an elective CS was recommended, with 5 patients $(1.9 \%)$ having no preferential recommendation and 1 patient $(0.4 \%)$ where a recommendation could not be found. An elective CS was recommended in 13 patients with complaints of fecal incontinence and an avulsion of the LAM, in 8 patients with fecal incontinence and a residual lesion of the IAS and/or the EAS, in 3 patients with fecal incontinence without residual lesions and in 37 patients with pelvic floor lesions (either LAM avulsions or sphincter lesions) without fecal incontinence. In the other ten patients an elective CS was recommended because of symptoms of fecal urgency ( 3 patients), severe shoulder dystocia at the index delivery (2), fear of recurrence (2), additional sphincter surgery after the initial OASIs surgery (1) and unclear reasons (2).

\section{Subsequent parturition mode and recurrent OASI}

Of the 264 patients that received a recommendation on the PM, 161 had a subsequent delivery during our study; 93 patients $(58 \%)$ had a VD and 68 patients $(42 \%)$ had a CS. In one patient a vacuum extraction was performed. All patients with the recommendation for an elective CS or no preferential recommendation on PM delivered by CS, in contrast to those who had a recommendation to aim for VD (91\% adherence). Seven patients (9\%) with the recommendation to aim for VD were delivered by CS on request because of fear of recurrence of OASIs. The recommendation to aim for VD with preconditions was not followed by two patients. One of them suffered from a recurrent OASI after the birth of an antenatally diagnosed macrosomic fetus (weight $>95$ th percentile for gestational age). The other had an elective CS. The remainder of the CSs in this group were performed for not meeting the preconditions, e.g., fetal macrosomia, worsening symptoms of incontinence and other maternal or fetal reasons. Adherence to the PM recommendation was significantly lower for VD recommendations compared with CS recommendations, $92 \%$ compared with $100 \%$, respectively, $p=0.036$ (Table 2).

A recurrence of OASIs occurred in $4(4.3 \%)$ of the 93 patients who had a subsequent VD. Three patients had a VD recommendation; one had a VD recommendation with preconditions and did not adhere to this as described above. In the subsequent delivery the episiotomy rate was $61 \%(33 / 54)$ in secondary care compared with 55\% (17/31) in primary care, $p=0.57$. In eight patients the episiotomy use was unknown.

\section{Symptoms of pelvic floor disorders within 5 years}

The questionnaire was returned by 183 out of 320 (57\%) patients. The median time between occurrence of OASIs and returning the questionnaire was 2.7 years. Symptoms were scored on a scale from 0 to 100 . The mean urinary incontinence score for the cohort (derived from the UDI) was 19. The 
Table 1 Baseline characteristics at the moment of the index delivery

Index delivery

$(\boldsymbol{n}=320)$

\begin{tabular}{|c|c|}
\hline \multicolumn{2}{|l|}{ Obstetric history } \\
\hline Parity 0 & $219(68.4 \%)$ \\
\hline Parity 1 & $91(28.4 \%)$ \\
\hline Parity 2 & $9(2.8 \%)$ \\
\hline Parity 3 & $1(0.3 \%)$ \\
\hline \multicolumn{2}{|l|}{ Delivery characteristics } \\
\hline Primary care & $111(34.7 \%)$ \\
\hline Secondary care & $205(65.3 \%)$ \\
\hline Home birth & $60(18.8 \%)$ \\
\hline Hospital birth & $260(81.3 \%)$ \\
\hline Gestational age; median (IQR) & $\begin{array}{l}\text { 40w1d } \\
\quad(39 w 0 d-40 w 6 d)\end{array}$ \\
\hline Birthweight; mean (min-max) & $3625 \mathrm{~g}(2188-5120)$ \\
\hline \multicolumn{2}{|l|}{ Presenting part } \\
\hline Occiput posterior & $37(11.6 \%)$ \\
\hline Occiput anterior & $283(88.4 \%)$ \\
\hline \multicolumn{2}{|l|}{ Operative vaginal delivery } \\
\hline None & $234(73.1 \%)$ \\
\hline Ventouse & $85(26.6 \%)$ \\
\hline Forceps & $1(0.3 \%)$ \\
\hline \multicolumn{2}{|l|}{ Episiotomy } \\
\hline No & $187(58.4 \%)$ \\
\hline Yes & $131(40.9 \%)$ \\
\hline Missing & $2(0.6 \%)$ \\
\hline \multicolumn{2}{|l|}{ OASI classification } \\
\hline Grade 3a & $80(25.0 \%)$ \\
\hline Grade $3 b$ & $197(61.6 \%)$ \\
\hline Grade 3c & $23(7.2 \%)$ \\
\hline Grade 4 & $18(5.6 \%)$ \\
\hline Missing & $2(0.6 \%)$ \\
\hline \multicolumn{2}{|l|}{ Clinic visit } \\
\hline Yes & $265(82.8 \%)$ \\
\hline No & $55(17.2 \%)$ \\
\hline $\begin{array}{l}\text { Time between OASIs and clinic visit in months; } \\
\text { median (IQR) }\end{array}$ & $6.2(5.8-8.3)$ \\
\hline $\begin{array}{l}\text { Time between OASIs and questionnaire in } \\
\text { months; } \\
\text { median (IQR) }\end{array}$ & $32.0(24.9-43.6)$ \\
\hline
\end{tabular}

Data presented as number of patients (\%) unless stated differently

mean fecal incontinence, flatus incontinence and pain related to defecation scores (derived from the DDI) were 1.2, 21 and 11 , respectively. The mean dyspareunia score derived from the questionnaire was 20 . The frequencies of anal incontinence symptoms are summarized in Table 3. Fecal incontinence occurring more than once a month was present in nine patients (4.9\%). With fecal incontinence occurring less than once a month added to this proportion, it was present in 26 patients $(14 \%)$. One hundred fourteen patients $(62 \%)$ had any form of urinary incontinence, $73(40 \%)$ had flatus incontinence and 75 $(41 \%)$ reported any form of dyspareunia $2-5$ years after OASIs. Using the St. Mark's score [16], 41 patients (22\%) had a score $>5$. An important factor in the St. Mark's score is the occurrence of fecal urgency, which 42 (23\%) of our patients were suffering from after OASIs. In total, 88 patients $(48 \%)$ in our cohort had any form of anal incontinence including fecal urgency, fecal and flatus incontinence occurring more than once a month.

\section{Pelvic floor injuries}

The integrity of the anal sphincter complex on 3D ultrasound was recorded in 248 cases. In total, 213 cases (86\%) had an intact internal anal sphincter (IAS) and EAS. In 26 patients (10\%), a defect of the EAS was noted. In five patients (2.0\%), an isolated defect of the IAS was seen and in four patients $(1.6 \%)$ a combined defect of both the IAS and EAS. Patients with a sphincter complex defect on ultrasound reported significantly more dyspareunia (33 vs. $19 ; p=0.04$ ). Moreover, they reported significantly more pain related to defecation ( 21 vs. $11 ; p=0.01$ ), and a non-significant trend towards more fecal incontinence ( 3.5 vs. $0.8 ; p=0.12$ ).

The integrity of the LAM on 3D ultrasound was recorded in 231 cases. In total, 174 patients $(75 \%)$ had no signs of avulsion. In 46 patients (20\%), a unilateral avulsion and in 11 patients $(4.7 \%)$ a bilateral avulsion of the LAM was diagnosed. Women with an avulsion of the LAM reported significantly more anal incontinence for solid or liquid stools (2.8 vs. $0.7 ; p=0.02$ ) and more dyspareunia ( 30 vs. $18 ; p=0.005$ ).

Women with grade 4 OASIs compared with grade $3 \mathrm{a}$ OASIs reported significantly more anal incontinence as measured by the St. Mark's fecal incontinence severity score ( 4.5 IQR $2.0-8.8$ vs. 1.0 IQR $1.0-4.0 ; p=0.009$ ).

\section{Discussion}

In this study we reported on practice in managing the mode of delivery after OASI in a large Dutch obstetric hospital. Our primary objective was to evaluate PM recommendations for subsequent deliveries following OASIs, and we found that in $71.2 \%$ and $26.9 \%$ of patients a VD or CS was recommended, respectively. This recommendation was followed by $94.4 \%$ of patients. Adherence to the PM recommendation was significantly lower for a recommendation of VD compared with CS with possible reasons being fear of recurrence of OASIs in a subsequent VD and traumatic experiences from the index VD. After a subsequent $\mathrm{VD}$, a recurrence of OASIs was observed in $4.3 \%$. After a median follow-up time of 2.7 years after OASI, $48 \%$ of patients reported any form of anal incontinence whereas fecal incontinence in solid or liquid form occurred in $4.9 \%$ of patients. 
Fig. 1 Flowchart of patient follow-up and mode of subsequent delivery

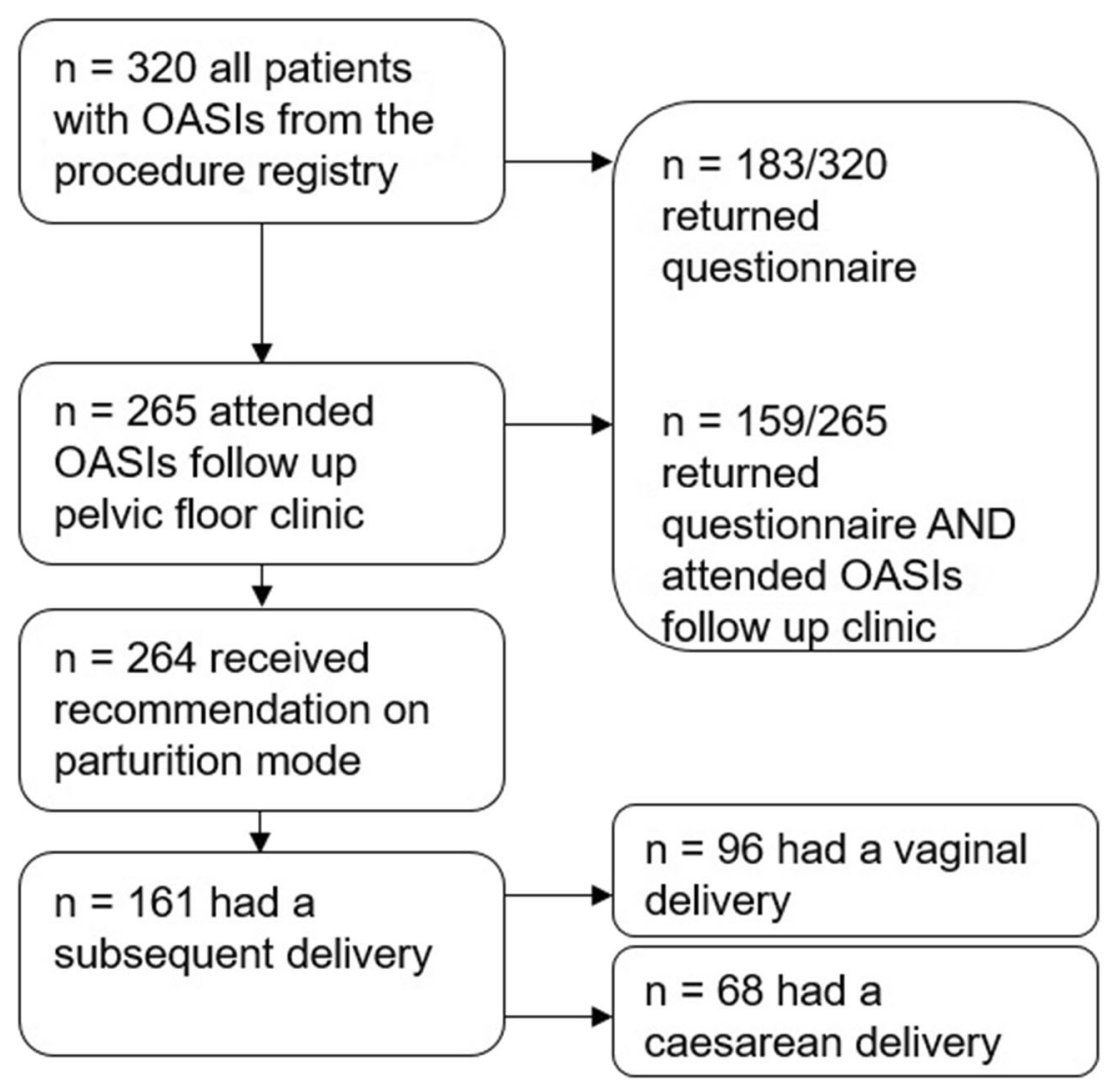

Our cohort is comparable to groups in earlier studies on this subject, with respect to vertex presentation, OVDs and subdivision between different classifications of OASIs [17]. To our knowledge, this is one of the biggest cohorts of patients that received a recommendation for a subsequent delivery [10]. More than $90 \%$ of patients adhered to the recommendation with only $9 / 161$ cases $(5.6 \%)$ not adhering, including one case in which a recurrence of OASI could have been prevented. The reason for not following the recommendations were traumatic experiences originating from the index delivery in the majority of cases. An additional eight CSs were therefore done.

Fifty-eight percent of patients had a subsequent VD and $42 \%$ of patients delivered by $\mathrm{CS}$, a figure much higher than

Table 2 Recommended parturition mode and adherence to the recommendation in a subsequent delivery

\begin{tabular}{lrl}
\hline Recommended parturition mode & $n$ & Adherence $n(\%)$ \\
\hline Vaginal delivery & 81 & $74(91 \%)$ \\
Vaginal delivery with preconditions & 29 & $27(93 \%)$ \\
Elective cesarean section & 48 & $48(100 \%)$ \\
No preference & 3 & $3(100 \%)$ \\
Total & 161 & \\
Recurrence of OASIs & 4 & $3(75 \%)$ \\
\hline
\end{tabular}

Number of patients the overall CS rate of just above $15 \%$ in The Netherlands [22]. However, the figure is similar to that of other studies on this subject $[17,23]$. The question is whether the higher morbidity in performing a CS outweighs the benefit of preventing eventual additional damage. Compared with previous studies [10, 17] with a reported rate of 3.6-10\%, our OASI recurrence rate $(4.3 \%)$ is within range, but it is still approximately double the index rate of $2.2 \%$.

All patients with fecal incontinence or residual pelvic floor or anal sphincter injuries should be counseled on the risks and benefits of a CS. Further pregnancy wishes and emotional sequelae must be taken into consideration, and patients must be informed that a recurrence of OASI is not always preventable.

Compared with endoanal ultrasound, which is the gold standard, 3D transperineal ultrasonography is an accepted method for detecting anal sphincter defects and is therefore a suitable screening method for residual lesions after OASI [24, 25]. Three-dimensional transperineal ultrasound compared with endoanal ultrasound had a $95 \%$ sensitivity and $75 \%$ specificity for the EAS and a $73 \%$ sensitivity and $93 \%$ specificity for the IAS [24]. The advantage is that the levator ani muscle (LAM) can be well represented with 3D transperineal ultrasound, and the scan is less invasive. An avulsion of the LAM may play a role in anal incontinence $[26,27]$. Little is known about the exact role of the LAM in anal incontinence after OASIs. It is known, however, that a lesion of the LAM after 
Table 3 Complaints of anal incontinence $2-5$ years after OASI repair

\begin{tabular}{|c|c|c|c|}
\hline Anal incontinence complaints & & $n=$ & $\%$ \\
\hline \multirow[t]{2}{*}{ Solid stool fecal incontinence* } & Less than once a month & 5 & 2.8 \\
\hline & More than once a month & 3 & 1.6 \\
\hline \multirow[t]{2}{*}{ Liquid stool fecal incontinence* } & Less than once a month & 18 & 9.8 \\
\hline & More than once a month & 8 & 4.4 \\
\hline \multirow[t]{2}{*}{ Flatus incontinence* } & Less than once a month & 36 & 19.7 \\
\hline & More than once a month & 73 & 39.9 \\
\hline Fecal urgency (defer less than $15 \mathrm{~min}$ )* & & 42 & 23.0 \\
\hline \multirow[t]{2}{*}{ Any form of anal incontinence } & Never or less than once a month & 95 & 51.9 \\
\hline & More than once a month & 88 & 48.1 \\
\hline \multirow[t]{3}{*}{ St. Mark's score } & $0-4$ & 142 & 77.6 \\
\hline & $5-8$ & 30 & 16.4 \\
\hline & 9 and above & 11 & 6.0 \\
\hline
\end{tabular}

*The remainder of patients in these categories reported complaints as never occurring

OASIs is found in $19 \%$ with MRI compared with $3.5 \%$ if no sphincter injury has occurred [27].

Pelvic floor injuries after OASI examined with 3D ultrasound are possible predictors for complaints of dyspareunia, anal pain and fecal incontinence. Most previous studies did not evaluate the role of the LAM in fecal incontinence after OASI [10, 17, 23]. In our cohort fecal incontinence was associated with avulsion of the LAM, a finding similar to a recent publication [28]. Both the EAS and the LAM are important for keeping fecal continence, and further research should be targeted to injuries of both anatomical structures after OASIs and their interaction in fecal incontinence.

The strength of this study is the extensive follow-up of all patients that received a recommendation on the PM and had a consecutive delivery. More than $60 \%$ of patients delivered again. We presented symptoms of pelvic floor disorders from validated questionnaires with a reasonable response rate of $57 \%$ 2-5 years after OASI and were able to correlate them to clinical findings 6 months after OASI when most temporary effects on the pelvic floor muscle function after pregnancy and childbirth would be expected to have resolved [29].

This study does contain some limitations, the most important being its retrospective design causing incomplete or missing data at baseline, and a significant loss to follow-up (see Fig. 1). This could have potentially introduced bias-for example, through underreporting of severe symptoms by patients that are traumatized, embarrassed or otherwise not wanting to be confronted with their symptoms. The lack of questionnaires at baseline prevented comparison of complaints before and after a consecutive delivery between VD and CS. The recommendation to deliver by $\mathrm{CS}$ was given more often to symptomatic women and is therefore an important confounder for symptoms after a consecutive delivery. Furthermore, 3D transperineal ultrasound volumes were not available for blinded review, and tomographic ultrasound imaging was not possible with the equipment used. The $86 \%$ of women without any sign of residual defect after OASIs is very high, and only 9 of the 41 women with a grade $3 \mathrm{c}$ or 4 OASIs had a defect visualized by transperineal ultrasound. Without the use of tomographic ultrasound imaging, transperineal ultrasound appears to have caused underreporting of residual sphincter lesions. The findings on pelvic floor injuries in this study must therefore be confirmed by further research.

In summary, this study showed that PM recommendations based on symptoms and pelvic floor injuries were followed by the vast majority of patients. This resulted in a low recurrence of OASIs but a high CS rate. Besides dyspareunia, urgency and flatus incontinence, which are common, fecal incontinence after OASIs occurred in $4.9 \%$. Fecal incontinence was related to the grade of OASI but possibly also to avulsion of the LAM. Further research investigating the role of avulsion of the LAM in fecal incontinence and potential interventions that could be used to reduce its rates is warranted.

Authors' contribution van der Vlist, M.Y.; Protocol/project development. Data collection. Data analysis. Manuscript writing/editing.

Oom, D.M.J.; Manuscript writing/editing.

van Rosmalen, J; Data analysis.

van Ovost, A.M.A; Data collection. Manuscript writing/editing.

Hogewoning, C.J.A.; Protocol/project development. Manuscript writing/editing.

\section{Compliance with ethical standards}

Conflict of interest None.

\section{References}

1. Gurol-Urganci I, Cromwell DA, Edozien LC, Mahmood TA, Adams EJ, Richmond DH, et al. Third- and fourth-degree perineal tears among primiparous women in England between 2000 and 2012: time trends and risk factors. BJOG. 2013;120(12):1516-25. 
2. Selmer-Olsen T, Nohr EA, Tappert C, Eggebo TM. Incidence and risk factors for obstetric anal sphincter ruptures, OASIS, following the introduction of preventive interventions. A retrospective cohort study from a Norwegian hospital 2012-2017. Sex Reprod Healthc. 2019;22:100460.

3. de Leeuw JW, Struijk PC, Vierhout ME, Wallenburg HC. Risk factors for third degree perineal ruptures during delivery. BJOG. 2001;108(4):383-7.

4. Farrar D, Tuffnell DJ, Ramage C. Interventions for women in subsequent pregnancies following obstetric anal sphincter injury to reduce the risk of recurrent injury and associated harms. Cochrane Database Syst Rev. 2014 (11):CD010374. doi(11):CD010374.

5. Ginath S, Mizrachi Y, Bar J, Condrea A, Kovo M. Obstetric Anal Sphincter Injuries (OASIs) in Israel: A Review of the Incidence and Risk Factors. Rambam Maimonides Med J. 2017 8(2):https://doi. org/10.5041/RMMJ.10295.

6. van Bavel J, Hukkelhoven CWPM, de Vries C, Papatsonis DNM, de Vogel J, Roovers JWR, et al. The effectiveness of mediolateral episiotomy in preventing obstetric anal sphincter injuries during operative vaginal delivery: a ten-year analysis of a national registry. Int Urogynecol J. 2018;29(3):407-13.

7. de Leeuw JW, de Wit C, Kuijken JP, Bruinse HW. Mediolateral episiotomy reduces the risk for anal sphincter injury during operative vaginal delivery. BJOG. 2008;115(1):104-8.

8. Sagi-Dain L, Sagi S. Morbidity associated with episiotomy in vacuum delivery: a systematic review and meta-analysis. BJOG. 2015;122(8):1073-81.

9. Meister MR, Cahill AG, Conner SN, Woolfolk CL, Lowder JL. Predicting obstetric anal sphincter injuries in a modern obstetric population. Am J Obstet Gynecol. 2016;215(3):310.e1-7.

10. Jordan PA, Naidu M, Thakar R, Sultan AH. Effect of subsequent vaginal delivery on bowel symptoms and anorectal function in women who sustained a previous obstetric anal sphincter injury. Int Urogynecol J. 2018;29(11):1579-88.

11. Mous M, Muller SA, de Leeuw JW. Long-term effects of anal sphincter rupture during vaginal delivery: faecal incontinence and sexual complaints. BJOG. 2008;115(2):234-8.

12. Sultan AH, Kamm MA, Hudson CN, Bartram CI. Third degree obstetric anal sphincter tears: risk factors and outcome of primary repair. BMJ. 1994;308(6933):887-91.

13. Borello-France D, Burgio KL, Richter HE, Zyczynski H, Fitzgerald MP, Whitehead W, et al. Fecal and urinary incontinence in primiparous women. Obstet Gynecol. 2006;108(4):863-72.

14. Andrews V, Shelmeridine S, Sultan AH, Thakar R. Anal and urinary incontinence 4 years after a vaginal delivery. Int Urogynecol J. 2013;24(1):55-60.

15. Jango H, Langhoff-Roos J, Rosthoj S, Sakse A. Recurrent obstetric anal sphincter injury and the risk of long-term anal incontinence. Am J Obstet Gynecol. 2017;216(6):610.e1-8.

16. Vaizey CJ, Carapeti E, Cahill JA, Kamm MA. Prospective comparison of faecal incontinence grading systems. Gut. 1999;44(1):7780 .
17. Vasseur A, Lepigeon K, Baud D, Horsch A, Meyer S, Vial Y, et al. Counseling after perineal laceration: does it improve functional outcome? Int Urogynecol J. 2019;30(6):925-31.

18. Third- and Fourth-degree Perineal Tears, Management (Green-top Guideline No. 29) [Internet].: Royal College of Obstetricians and Gynaecologists; 2015 [updated 12.06.2015; cited 29.10.2019]. Available from: https://www.rcog.org.uk/en/guidelines-researchservices/guidelines/gtg29/.

19. Shumaker SA, Wyman JF, Uebersax JS, McClish D, Fantl JA. Health-related quality of life measures for women with urinary incontinence: the incontinence impact questionnaire and the urogenital distress inventory. Continence program in women (CPW) research group. Qual Life Res. 1994;3(5):291-306.

20. van der Vaart CH, de Leeuw JR, Roovers JP, Heintz AP. Measuring health-related quality of life in women with urogenital dysfunction: the urogenital distress inventory and incontinence impact questionnaire revisited. Neurourol Urodyn. 2003;22(2):97-104.

21. Roovers JP, van der Bom JG, van der Vaart CH, Heintz AP. Prediction of findings at defecography in patients with genital prolapse. BJOG. 2005;112(11):1547-53.

22. Zhang J, Geerts C, Hukkelhoven C, Offerhaus P, Zwart J, de Jonge A. Caesarean section rates in subgroups of women and perinatal outcomes. BJOG. 2016;123(5):754-61.

23. Karmarkar R, Bhide A, Digesu A, Khullar V, Fernando R. Mode of delivery after obstetric anal sphincter injury. Eur J Obstet Gynecol Reprod Biol. 2015;194:7-10.

24. Ros C, Martinez-Franco E, Wozniak MM, Cassado J, Santoro GA, Elias N, et al. Postpartum two- and three-dimensional ultrasound evaluation of anal sphincter complex in women with obstetric anal sphincter injury. Ultrasound Obstet Gynecol. 2017;49(4):508-14.

25. Oom DM, West RL, Schouten WR, Steensma AB. Detection of anal sphincter defects in female patients with fecal incontinence: a comparison of 3-dimensional transperineal ultrasound and 2dimensional endoanal ultrasound. Dis Colon Rectum. 2012;55(6): 646-52.

26. Tankova L, Draganov V, Damyanov N. Endosonography for assessment of anorectal changes in patients with fecal incontinence. Eur J Ultrasound. 2001;12(3):221-5.

27. Heilbrun ME, Nygaard IE, Lockhart ME, Richter HE, Brown MB, Kenton KS, et al. Correlation between levator ani muscle injuries on magnetic resonance imaging and fecal incontinence, pelvic organ prolapse, and urinary incontinence in primiparous women. Am J Obstet Gynecol. 2010;202(5):488.e1-6.

28. Martinez Franco E, Lopez Negre JL, Pares D, Ros Cerro C, Amat Tardiu L, Cuadras D, et al. Anatomic and functional evaluation of the levator ani muscle after an obstetric anal sphincter injury. Arch Gynecol Obstet. 2019;299(4):1001-6.

29. Elenskaia K, Thakar R, Sultan AH, Scheer I, Beggs A. The effect of pregnancy and childbirth on pelvic floor muscle function. Int Urogynecol J. 2011;22(11):1421-7.

Publisher's note Springer Nature remains neutral with regard to jurisdictional claims in published maps and institutional affiliations. 